\title{
Sample introduction in multi-syringe flow injection systems: comparison between time-based and volume-based strategies
}

\author{
Marcela A. Segundo ${ }^{\text {a } *}$, Hugo M. Oliveira ${ }^{\text {a }}$, José L.F.C. Lima ${ }^{a}$, \\ M. Inês G.S. Almeida ${ }^{\mathrm{b}}$, António O.S.S. Rangel ${ }^{\mathrm{b}}$ \\ a REQUIMTE, Departamento de Química-Física, Faculdade de Farmácia, Universidade do Porto, Rua Aníbal Cunha, 164, 4099-030 Porto, Portugal \\ ${ }^{\mathrm{b}}$ Escola Superior de Biotecnologia, Universidade Católica Portuguesa, Rua Dr. António Bernardino de Almeida, 4200-072 Porto, Portugal
}

Keywords: Multi-syringe; Flow injection; Sampling strategy; Time-based sampling; Volume-based sampling

\begin{abstract}
In multi-syringe flow injection analysis (MSFIA), devices as selection, injection or commutation valves must be incorporated to the manifold to provide access to sample and standard solutions. Therefore, the definition of sample amount can be either volume or time-based. In the present work, four configurations for sample introduction (two for each approach) were tested in order to establish if the different strategies affect the analytical signal in MSFIA systems. The mean absorbance value from ten consecutive injections of a bromothymol blue solution obtained for the time-based strategy was lower than that provided by the volume-based approach as the exact volume delivered by each configuration was different from the "theoretical" volume. For time-based configurations, the exact volume delivered is $2-5 \%$ lower than the theoretical value while for volume-based configurations, the volume delivered was between 6 and $46 \%$ larger than the theoretical volume. Moreover, for time-based sampling, the order of steps in the analytical cycle was of utmost importance since any alteration in the flow direction affected the volume delivered in the subsequent step in the analytical cycle. The influence of the two sampling approaches was also evaluated in the MSFIA systems for the spectrophotometric determination of phenolic compounds and the potentiometric determination of chloride. There was no evidence that the use of either volume or time-based sampling would improve the analytical features of these determinations when real samples were tested.
\end{abstract}

\section{Introduction}

Multi-syringe flow injection analysis (MSFIA) was introduced by Cerdà et al. in 1999 [1] as a robust alternative to its predecessor techniques, especially when applications employing organic solvents are considered [2]. The features of these flow systems allow the assembly of a flow network, connected to a detection system, where reagents from the different syringes can be either delivered to the flow system or returned to its own vessel, without interfering with the other channels.

\footnotetext{
* Corresponding author. Tel.: +351 22 2078994; fax: +351 222004427.

E-mail address: msegundo@mail.ff.up.pt (M.A. Segundo).
}

In this type of flow systems it is not feasible to introduce the sample into the system by filling one of the available syringes as it would take a long time of washing steps for avoiding carry-over between consecutive samples [3]. Moreover, the amount of sample required for those washing steps would be considerably large, which is not compatible with samples that are scarce or expensive.

Therefore, other devices as selection [4], injection [5,6] or commutation [7-9] valves must be incorporated to the manifold to provide access to sample and standard solutions. Two different strategies can be used to define the sample amount: volume-based and time-based strategies [10]. The volumebased approach is based on the entrapment of a certain volume in a well-defined tube portion, which is subsequently pushed by carrier. The time-based mode relies on the aspiration or 
propulsion of a certain volume, defined by the flow rate and the time during which the pumping device is activated.

The main objective of this work was to establish if the different strategies affect the analytical signal in MSFIA systems. Therefore, different configurations for sample introduction using both strategies were tried, using a colored solution to evaluate the effect on the analytical signal. Moreover, the influence of these two approaches in MSFIA systems already described was evaluated. For this purpose, the spectrophotometric determination of phenolic compounds [11] and the potentiometric determination of chloride [12] were chosen.

\section{Materials and methods}

\section{Reagents and solutions}

Water from MilliQ system (resistivity > $18 \mathrm{M} \Omega \mathrm{cm}$ ) was used for the preparation of all solutions, and all chemicals were analytical-reagent grade.

For assessment of the influence of different strategies in the analytical signal, a bromothymol blue (BTB) solution was prepared from a stock solution $\left(0.50 \mathrm{~g}^{-1}\right)$ by dilution in $0.010 \mathrm{moll}^{-1}$ borax solution in order to provide an absorbance value of $0.725 \pm 0.005$ at $620 \mathrm{~nm}$.

For the determination of the exact volume delivered using the different strategies, $0.5 \mathrm{~mol}^{-1} \mathrm{HCl}$ standard solution (Titrisol $^{\circledR}$, Merck, Darmstadt), $\mathrm{NaOH}\left(0.08 \mathrm{moll}^{-1}\right)$ solution and phenolphthalein indicator solution were used.

For the determination of total phenolics the following solutions were prepared as described by Oliveira [11]: $\mathrm{NaOH}\left(0.10 \mathrm{~mol} \mathrm{l}^{-1}\right) ; \mathrm{K}_{3}\left[\mathrm{Fe}(\mathrm{CN})_{6}\right]\left(1.50 \mathrm{~g} \mathrm{l}^{-1}\right)$; buffer solution containing $0.124 \mathrm{moll}^{-1}$ of $\mathrm{H}_{3} \mathrm{BO}_{3}$ and $\mathrm{pH}$ adjusted to 8.0 by addition of concentrated $\mathrm{NaOH}$ solution. The 4aminoantipyrine was dissolved in buffer solution $\left(0.50 \mathrm{~g}^{-1}\right)$. The working standards were prepared from a stock solution containing $1.00 \mathrm{~g}^{-1}$ of phenol. The reference material was obtained from LGC Promochem (Middlesex, UK).

For the determination of chloride, the solutions were prepared as indicated by Andrade-Eiroa et al. [12], except for the ionic strength adjuster solution (ISA), containing $0.05 \mathrm{moll}^{-1} \mathrm{NaNO}_{3}$ and $1 \mathrm{mg} \mathrm{l}^{-1}$ chloride.

\section{Apparatus}

The Crison multi-syringe burettes (Allela, Spain) used in the present work were multiple channel piston pump, where all pistons were driven by a single motor, controlled by computer software through a serial port. A three-way commutation valve (NResearch, Caldwell, NJ, USA) was connected to the head of each syringe. For all valves, the exchange options were classified in on/off lines.

The piston displacement from the lowest to the highest position was divided in a fixed number of steps, which defined the minimum volume to be delivered (that also depended on the syringe capacity). In the present work, two multi-syringe burettes were used, where the piston movement was divided in 5000 and 16000 steps, according to the model applied. Hence, when a $5.00 \mathrm{ml}$ syringe was applied, the minimum volume delivered was about 1 and $0.3 \mu l$, respectively.

For sample introduction, additional commutation valves from NResearch with internal volume of $27 \mu$ l (ref. 161T031) and $57 \mu \mathrm{l}$ (ref. HP225T031) were introduced in the flow system. Laboratory-made acrylic Y-shaped connectors were used as confluences. The length of the inner channels was $15 \mathrm{~mm}$.

The connections and sample loops were made of Omnifit (Cambridge, UK) PTFE tubing ( $0.8 \mathrm{~mm}$ i.d.) with Gilson (Villiers-le-Bel, France) end-fittings and connectors.

A personal computer, running lab-made software written in QuickBasic 4.5 (Microsoft), controlled the multi-syringe operation (number of steps and direction of piston displacement and position of all commutation valves).

For the spectrophotometric measurements, a Jenway 6300 (Essex, UK) spectrophotometer equipped with a Hellma (Mullheim/Baden, Germany) 178.710-QS flow-through cell (internal volume $80 \mu \mathrm{l}$ ) was used and the wavelength was set at $620 \mathrm{~nm}$ (experiments using BTB) or $510 \mathrm{~nm}$ (determination of total phenolics).

For the potentiometric measurements, a millivoltmeter (Crison, model 2002) and a double-junction reference electrode (Russel, model 90-0029), with a $0.1 \mathrm{moll}^{-1} \mathrm{KNO}_{3}$ solution in the outer compartment were used. As indicator electrode, a homogenous crystalline membrane chloride ionselective electrode without inner reference solution and with a tubular configuration [13] was used.

For both detection systems, data acquisition was performed through a PCL-818L interface card at $4 \mathrm{~Hz}$, using the same software developed for controlling the flow system. The data obtained was analyzed using either Microsoft Excel 2002 or Origin 6.1 software.

\section{Configurations adopted for sampling}

Different configurations for sample introduction were selected for comparing volume-based (Fig. 1A and B) and timebased (Fig. 1C and D) strategies.

Using configuration A, sample was aspirated through the sample loop by means of syringe 1 with valves 5 and 6 in position off. After commutation of the solenoid valves, the sample portion entrapped between the two valves was pushed towards the flow network by action of syringe 2 .

Configuration B is simpler than configuration A since it implies a similar operation for sample introduction using only one valve. In this case, the principle behind hydrodynamic injection [14] was applied as the sample loop was connected to three channels at the same time and one of them was filled by a stagnant liquid. This column of liquid exerted a hydrostatic force, which acted as a lock while the sample loop was filled or washed by carrier.

Both configurations $\mathrm{C}$ and $\mathrm{D}$ rely on time-based sampling. In configuration $\mathrm{C}$, a sample amount defined by the time 

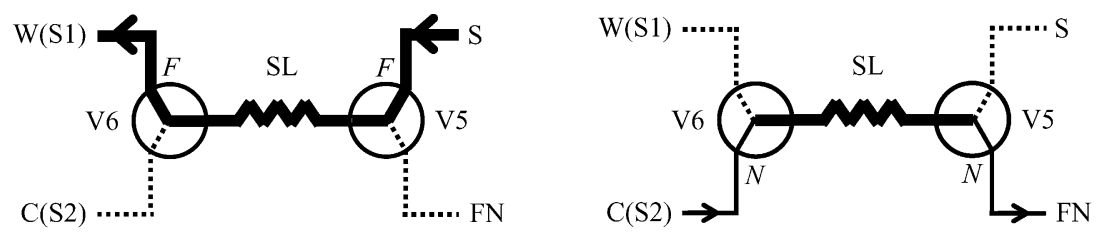

Configuration A
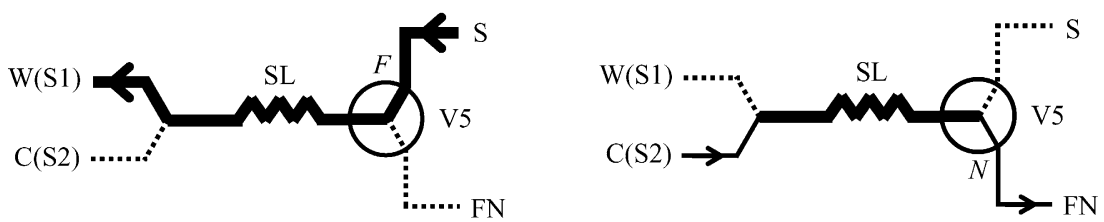

Configuration B
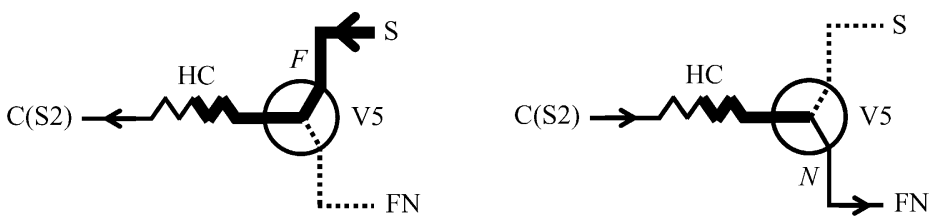

Configuration C
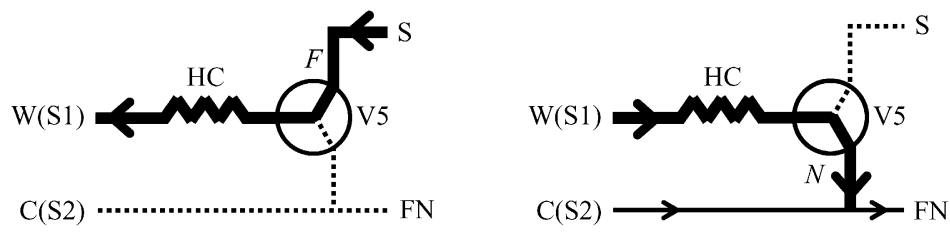

Configuration D

Fig. 1. Configurations adopted for sample introduction using volume-based (A, B) and time-based (C, D) strategies, including schematic representations of sample uptake and introduction into the flow network. The solid, bold and dotted lines represent the channels filled with carrier, sample and stagnant liquid, respectively. $N$ and $F$ represent positions "on" and "off" in the additional valves (V5 and V6). S, sample; FN, flow network; SL, sample loop; W(S1), waste in syringe $1 ; \mathrm{C}(\mathrm{S} 2)$, carrier in syringe 2 ; $\mathrm{HC}$, holding coil.

and flow rate of aspiration was drawn into the holding coil (HC). By flow reversal, this amount was pushed by carrier towards the flow network. In configuration D, a larger amount of sample was aspirated to fill the $\mathrm{HC}$, reaching syringe 1 that worked as waste reservoir. In the next step, an aliquot of the sample contained in the $\mathrm{HC}$ was sent through a confluence point towards the flow network. Its volume was also defined by the time and flow rate of propulsion. Next, the sample plug was propelled further by carrier from syringe 2 . These two last steps could be performed twice before filling the syringes again.

In order to evaluate the influence of the different configurations in the analytical signal without reaction, the tubing addressed as "flow network" in Fig. 1 was composed by a coiled section $(l=50 \mathrm{~cm})$ plus a straight section $(l=50 \mathrm{~cm})$, connecting the injection module to the flow cell inside the spectrophotometer. BTB solution was injected 10 times consecutively in a $0.010 \mathrm{~mol}^{-1}$ borax carrier and the analytical signal profile (peak height and time at peak maximum) was determined.

For configurations $\mathrm{A}$ and $\mathrm{B}$, the sample loop was composed of PTFE tubing with $10 \mathrm{~cm}(50 \mu \mathrm{l}), 20 \mathrm{~cm}(100 \mu \mathrm{l})$ and $40 \mathrm{~cm}(200 \mu \mathrm{l})$. These "theoretical" volumes were calculated from the equation $S_{\mathrm{V}}=\pi R^{2} L$, where $S_{\mathrm{v}}$ is the sample volume, $R$ the internal radius of sample loop and $L$ the respective length. 
In order to determine the exact volume delivered by each configuration, a $0.5 \mathrm{~mol}^{-1} \mathrm{HCl}$ standard solution was used as sample and injected 10 times in a water carrier. The effluent of the flow network was titrated against standardized $\mathrm{NaOH}$.

\section{MSFIA systems for determination of chloride and total phenolic compounds}

The MSFIA system used for the determination of total phenolic compounds [11] is presented schematically in Fig. 2(I). The analytical cycle comprised three steps. In the first step, the syringes were filled with their respective solutions. Next, the sampling step was performed: for configuration $\mathrm{A}, 500 \mu \mathrm{l}$ of sample were aspirated through the sample loop while for configuration $\mathrm{C}, 100 \mu \mathrm{l}$ was aspirated towards the holding coil. In the last step, the sample was propelled by $\mathrm{NaOH}$ solution and was mixed sequentially with the buffer/4aminoantipyrine solution, followed by the $\mathrm{K}_{3}\left[\mathrm{Fe}(\mathrm{CN})_{6}\right]$ solution. The steps of this analytical cycle were performed at $7.50,2.00$ and $7.50(3 \times 2.50) \mathrm{ml} \mathrm{min}^{-1}$, respectively.
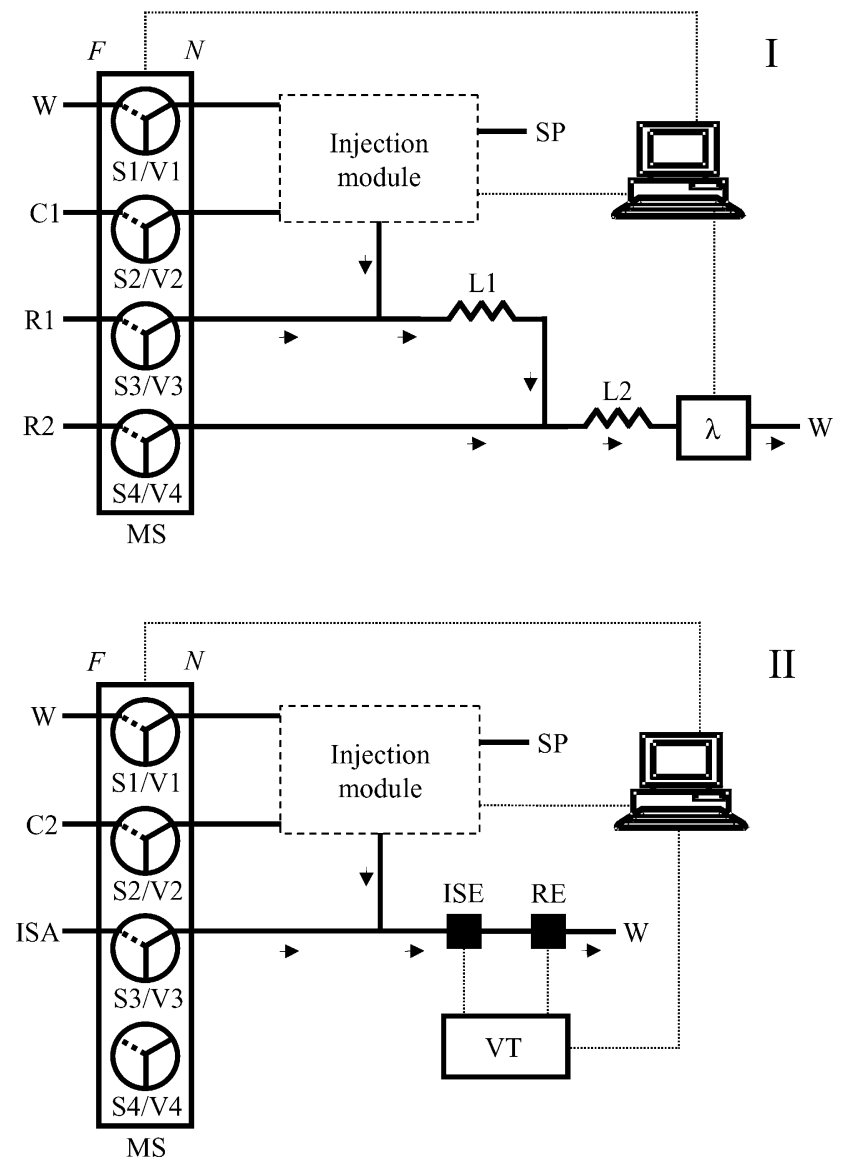

Fig. 2. MSFIA manifolds for the determination of total phenolic compounds (I) and chloride (II): MS, multi-syringe; $\mathrm{Si}$, syringe; Vi, commutation valves; $N$, on position (solid line); $F$, off position (dotted line); $\lambda$, spectrophotometer; ISE, ion selective electrode; RE, reference electrode; VT, millivoltmeter; $\mathrm{Li}$, PTFE connections $(\mathrm{L} 1=40 \mathrm{~cm} ; \mathrm{L} 2=105 \mathrm{~cm}) ; \mathrm{C} 1,0.10 \mathrm{~mol}^{-1} \mathrm{NaOH}$; R1, 4-aminoantipyrine/buffer solution; $\mathrm{R} 2,1.50 \mathrm{~g} \mathrm{l}^{-1} \mathrm{~K}_{3}\left[\mathrm{Fe}(\mathrm{CN})_{6}\right]$; C2, water; ISA, $0.05 \mathrm{moll}^{-1} \mathrm{NaNO}_{3}+1 \mathrm{mg} \mathrm{l}^{-1} \mathrm{Cl}^{-}$; SP, sample; W, waste.
In the potentiometric determination of chloride, the flow manifold proposed by Andrade-Eiroa et al. [12] was modified (Fig. 2II), since the ISA solution was only present in one of the flow channels. The analytical cycle was also composed of three steps and the first two were similar to those described for the spectrophotometric determination, including the flow rate. In the last step, the sample is pushed by water and subsequently mixed with ISA solution before reaching the detector. This step was performed at $4.50(3.00+1.50) \mathrm{ml} \mathrm{min}^{-1}$ as the capacity of all syringes was $5.00 \mathrm{ml}$, except for the ISA solution that was $2.50 \mathrm{ml}$.

\section{Results and discussion}

\section{General considerations about the operating conditions using the two strategies}

The implementation of volume or time-based sampling in MSFIA systems requires the introduction of different devices and/or different configurations in the flow manifold, as depicted in Fig. 1. As a consequence, the sampling steps in the analytical cycle can take more or less time according to the configuration chosen. This fact is particularly relevant when it is necessary to perform the change to the next sample to be analyzed. In this case, when volume-based configurations are used (Fig. 1A and B), no extra-operation is necessary since the uptake of the next sample can be performed at the same time as the loop is filled, as long as the volume drawn is enough to rinse all tubing containing the previous sample.

For the time-based approach, when configuration D is applied, the aspirated volume of the new sample has to be sufficiently large to avoid contamination of the last aliquot to be used. When configuration $\mathrm{C}$ is used, sample changing is more complex and takes more time since it is necessary to fill the sampling tube with the new sample and wash the $\mathrm{HC}$ with carrier before starting the analytical cycle. This configuration usually comprises another commutation valve that directs the flow towards waste, without passing through the flow network or detection system.

Volume-based sampling presents other advantages when compared to the time-based approach. As volume metering does not rely on the flow rate of aspiration/propulsion, it can be less affected by the physical properties of sample (viscosity, surface tension) when large flow rates are applied. For the same reason, the presence of air bubbles can affect significantly the time-based sampling while it is not important in volume-based sampling as long as they do not remain trapped inside the sample loop.

Nevertheless, the MSFIA systems presented in the literature evolved from volume-based to time-based sampling. In fact, the volume-based approach is only applied in a few of the first systems described. Despite the advantages presented before, the time-based approach is far more flexible, since any alteration in the sample volume can be performed through software control, without physical change required 
by volume-based configurations. The alteration of sample volume through software control enabled the application of a dynamic concentration range in the determination of warfarin [15] and the development of an expert system for the determination of iron [16], where the analyzed volume was adjusted to the concentration present in the sample.

When configurations $\mathrm{C}$ and $\mathrm{D}$ for time-based sampling are compared, the first one is more adequate when scarce samples (such as biological fluids) are analyzed since only the required volume is aspirated into the flow system. On the other hand, if the sample availability is not an issue, configuration D seems more suitable as it is possible to perform several determinations using aliquots of the same sample drawn. This feature can enhance the determination throughput as it is not necessary to refill the syringes between each determination.

\section{Precision and accuracy of volume delivery}

As the objective of this work was the comparison between volume-based and time-based strategies for sample introduction in MSFIA systems, four configurations for sample introduction were used (two for each approach) and the peak maximum values were determined for ten consecutive injections of a BTB solution using "theoretical" volumes of 50, 100 and $200 \mu \mathrm{l}$ (Table 1). For configurations A and B, the volume was defined by the radius and length of the sample tubing used as sample loop. For configurations C and D, the sample volume was defined by the software instruction given to the multi-syringe, comprising the number of steps and the velocity for displacement of the bar connected to the pistons. For all cases, the sampling step was performed at $2 \mathrm{ml} \mathrm{min}^{-1}$. The exact volume introduced in the flow system was also determined experimentally (Table 2 ).

Except for the volume of $200 \mu l$, the analytical signal obtained for the time-based strategy was lower than that provided by the volume-based approach. In fact, the exact volume delivered by each configuration was different from the theoretical volume. For configurations $\mathrm{C}$ and $\mathrm{D}$, the exact volume delivered was $2-5 \%$ lower than the theoretical value while for configurations $\mathrm{A}$ and $\mathrm{B}$, the volume delivered was between 6 and $46 \%$ larger than the theoretical volume. In this case, there was a positive deviation of about 18-23 $\mu$ l for configuration $\mathrm{A}$ and 11-15 $\mu$ l for configuration $\mathrm{B}$. These deviations were probably due to the contribution of the internal volume of the valves and the confluence used in both configurations.

This possibility was confirmed when the commutation valves were replaced by others with larger internal volume (57 $\mu \mathrm{l}$ instead of $27 \mu \mathrm{l}$ ). When larger valves were used, the analytical signal was about 0.020 absorbance units higher for configuration $\mathrm{A}$ and $\mathrm{B}$ while no alteration was verified for configuration $\mathrm{C}$. Moreover, for a theoretical volume of $100 \mu \mathrm{l}$, the exact volume delivered was about $10 \mu \mathrm{l}$ larger than that obtained using smaller valves, for both volumebased configurations.

Nevertheless, when $200 \mu \mathrm{l}$ were used, the analytical signal for configurations A, B and D were similar (Table 1), despite the differences in the exact volume delivered (217, 211 and 200, respectively). This situation was probably due to a lower effect of the constant dead volumes of valves when larger sample volumes were applied. In fact, the exact volume delivered for the volume-based configurations was between 10 and $50 \%$ larger than that obtained by configuration D for lower sample volumes (50 and $100 \mu \mathrm{l})$.

The differences in the analytical signals obtained using the two time-based configurations cannot be explained by those features. Since the exact volume delivered was similar, they are probably due to the fact that for configuration $\mathrm{C}$, only the amount of sample to be delivered was taken into the holding coil and subsequently propelled by carrier to the flow network. During these two steps the sample plug was dispersed in the carrier stream while for configuration $\mathrm{D}$, the sampling step did not involve dispersion in the carrier since a large amount of sample was drawn into the holding coil.

\section{Order of operations in the analytical cycle}

Previous work indicated that the order in which the different operations were carried out within the analytical cycle would affect the analytical signal whenever there was a change in the flow direction. In fact, Armas et al. [17] have reported that a "dummy" step was introduced in the analyti-

Table 1

Mean absorbance values at peak maximum $(n=10)$ for the same BTB solution using different configurations $(\mathrm{A}-\mathrm{D})$ in the injection module

\begin{tabular}{|c|c|c|c|c|}
\hline Theoretical volume $(\mu \mathrm{l})$ & A & B & $\mathrm{C}$ & $\mathrm{D}$ \\
\hline 50 & $0.106 \pm 0.001$ & $0.113 \pm 0.001$ & $0.071 \pm 0.002$ & $0.082 \pm 0.001$ \\
\hline 100 & $0.181 \pm 0.002$ & $0.189 \pm 0.001$ & $0.147 \pm 0.003$ & $0.161 \pm 0.002$ \\
\hline 200 & $0.308 \pm 0.003$ & $0.306 \pm 0.002$ & $0.288 \pm 0.002$ & $0.306 \pm 0.002$ \\
\hline
\end{tabular}

Table 2

Mean values $(n=10)$ of the volume introduced in the flow network using different configurations $(\mathrm{A}-\mathrm{D})$ in the injection module

\begin{tabular}{|c|c|c|c|c|}
\hline Theoretical volume $(\mu \mathrm{l})$ & A & B & $\mathrm{C}$ & $\mathrm{D}$ \\
\hline 50 & $73.2 \pm 0.7(+23.2)$ & $64.9 \pm 0.4(+14.9)$ & $47.5 \pm 0.3(-2.5)$ & $48.6 \pm 0.5(-1.4)$ \\
\hline 100 & $118 \pm 1(+18)$ & $111 \pm 1(+11)$ & $94.7 \pm 0.1(-5.3)$ & $98.3 \pm 0.3(-1.7)$ \\
\hline 200 & $217 \pm 1(+17)$ & $211 \pm 1(+11)$ & $195 \pm 1(-5)$ & $200 \pm 1(0)$ \\
\hline
\end{tabular}

The values in parenthesis are the absolute deviation values from the theoretical values. 
Table 3

Values of maximum absorbance and respective time after injection obtained using time-based strategy (configuration C) for different analytical cycles

\begin{tabular}{llll}
\hline Step order in analytical cycle & Movement direction & & \multicolumn{2}{c}{ Time (s) } \\
\cline { 2 - 3 } & Prior to sample aspiration & Prior to detection \\
\hline $1 / 2 / 3$ & $=$ & $\neq$ & $0.154 \pm 0.002$ \\
$2 / 1 / 3$ & $\neq$ & $\neq$ & $0.066 \pm 0.001$ \\
$2 / 3 / 1$ & $=$ & $=$ & $0.154 \pm 0.002$ \\
$1 / 2 / \mathrm{D} / 3$ & $=$ & $\neq$ & $0.152 \pm 0.002$ \\
$1 / \mathrm{D} / 2 / 3$ & $\neq$ & $0.061 \pm 0.003$ & $22.9 \pm 0.6$ \\
\hline
\end{tabular}

The steps are: (1) syringe filling; (2) sample aspiration; (3) propulsion to detector; (D) dummy step to change the movement direction. The sample volume was $100 \mu l$.

cal cycle to "avoid the loss of motor steps due to directional change of the syringe pistons when small volumes are to be handled". For this reason some experiments were carried out using the proposed configurations to evaluate how the change of flow direction would affect the analytical signal.

For configurations A, B and C, the analytical cycle was divided in three steps: (1) syringe filling (downward movement); (2) sample aspiration (downward movement); and (3) propulsion to the flow network/detector (upward movement).

When the order of steps 1 and 2 in the analytical cycle was changed (analytical cycle: $2 / 1 / 3$ ) there was no difference in the analytical signal obtained using configurations A or B for 10 consecutive injections of the BTB solution. Instead, for configuration $\mathrm{C}$, the maximum absorbance value was reduced to about one third of the initial value (Table 3 ).

To further investigate if the change of flow direction before the sampling step was behind the reduction of analytical signal, the order of steps in the analytical cycle was changed once again. Hence, in cycle $2 / 3 / 1$, there was not change in the flow direction and, despite the aspiration steps were not consecutive, the analytical signal was the same as for analytical cycle $1 / 2 / 3$. Moreover, when a "dummy" step was introduced between steps 1 and 2 in order to change the flow direction before sampling, the analytical signal was also lower when compared to that obtained for $1 / 2 / 3$ cycle.

These results provided evidence that the change of flow direction before sampling using configuration $\mathrm{C}$ affect the exact volume aspirated. To investigate if this situation was caused by mechanical reasons due to loss of steps during the change of direction of piston movement, the determination of the analytical signal and the exact volume delivered was performed using multi-syringes with the total piston displacement divided in 5000 or 16000 steps.

Two analytical cycles were tried (1/2/3 and $2 / 1 / 3)$ for theoretical volumes between 50 and $250 \mu \mathrm{l}$ (Figs. 3 and 4). From the absorbance values presented in Fig. 3, there is a systematic deviation when the flow direction is changed before sampling. This effect is more pronounced for the 5000 steps multi-syringe and it is probably due to the lower volume drawn when this device is applied (Fig. 4), even for volumes up to $200 \mu$ l.

The change of flow direction affects not only the aspiration but also the propulsion of solutions. This can be verified by comparing the results obtained by the analytical cycle com-

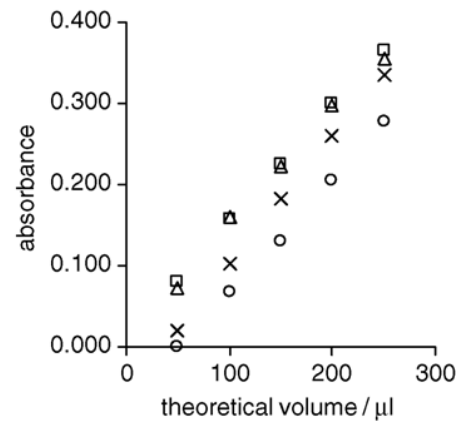

Fig. 3. Mean absorbance values at peak maximum $(n=10)$ obtained using configuration $\mathrm{C}$ when the step before sampling was performed in the same direction ( $\triangle$, MS with 5000 steps; $\square$, MS with 16000 steps) or in the opposite direction ( $\bigcirc$, MS with 5000 steps; $\times$, MS with 16000 steps).

posed by steps $1 / 2 / 3$ to those provided by a similar cycle with a "dummy" step (upward movement) placed between steps 2 and 3 to change the flow direction. The analytical signals were similar but the time of peak maximum differed in about $2.1 \mathrm{~s}$. For the first cycle (without "dummy" step), it took more time to achieve the maximum absorbance, which was caused by the delay in the piston movement due to the loss of steps.

For configuration D, the syringe filling (1) and sample aspiration (2) were performed simultaneously. In this case, the steps of sample introduction into the flow network and further propulsion towards the detector were performed twice

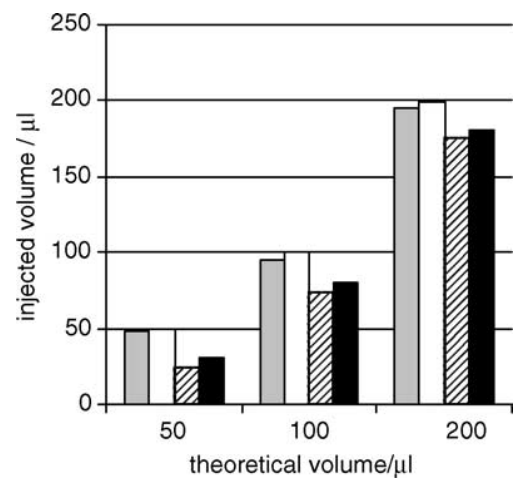

Fig. 4. Relation between the injected and the theoretical volume using configuration $\mathrm{C}$ when the step before sampling was performed in the same direction (grey bar, MS with 5000 steps; white bar, MS with 16000 steps) or in the opposite direction (bar with diagonal lines, MS with 5000 steps; black bar, MS with 16000 steps). 
Table 4

Figures of merit for the determination of total phenolic compounds using volume-based (A) and time-based (C) strategies for sample introduction

\begin{tabular}{lll}
\hline & Configuration A & Configuration C \\
\hline Calibration parameters & $\mathrm{AU}=0.0234 \pm 0.0001, \mathrm{C}+0.008 \pm 0.001, R=0.99995$ & $\mathrm{AU}=0.0195 \pm 0.0001, \mathrm{C}+0.005 \pm 0.001, R=0.9998$ \\
Limit of detection $\left(\mathrm{mg} \mathrm{l}^{-1}\right)$ & 0.17 & 0.34 \\
Determination frequency $\left(\mathrm{h}^{-1}\right)$ & 70 & 85 \\
Repeatability (RSD, $n=5)$ & $<1.3 \%$ & $<2.5 \%$ \\
Application to sample analysis (concentration in mg l & \\
QCI-043-1 $(10 \pm 1.53)$ & $8.85 \pm 0.09$ & $8.87 \pm 0.08$ \\
QCI-043-2 $(5.01 \pm 0.757)$ & $5.38 \pm 0.07$ & $5.39 \pm 0.06$ \\
Spiked tap water $(+2.50)$ & $2.56 \pm 0.04$ & $2.63 \pm 0.06$ \\
\hline
\end{tabular}

All concentration values are expressed in $\mathrm{mgl}^{-1}$.

before filling the syringes again, originating two peaks for each analytical cycle. When five consecutive analytical cycles were performed in this way, the first peak was always lower $(0.056 \pm 0.001, n=5)$ than the second one $(0.159 \pm 0.002$, $n=5)$. In order to change the flow direction between the syringe filling and the first step for introduction of sample aliquot, a "dummy" step where the solutions were directed towards their reservoir (upward movement) was introduced. In this case, the peaks were similar $(0.161 \pm 0.002, n=10)$ to the second peak of the previous analytical cycle. Therefore, the introduction of a "dummy" step is mandatory to avoid systematic error in the first analytical signal when using this configuration.

\section{Application of time-based and volume-based}

strategies in the same analytical determination

The multi-syringe flow systems described for the determination of total phenolic compounds and for chloride were chosen to study the influence of sampling strategy in the analytical features. These two determinations were chosen because they are based in different types of detection system and the systems previously described were based on either volume-based or time-based sampling.

The analytical features obtained when using configurations $\mathrm{A}$ and $\mathrm{C}$ for sample introduction (theoretical volume of $100 \mu \mathrm{l}$ ) are given in Tables 4 and 5, including the calibration curve parameters, limit of detection, determination frequency and precision.

For determination of phenolic compounds, the accuracy was assessed by analyzing certified reference material and spiked sample while the detection limit was calculated using the calculated intercept as an estimate of the blank signal and the statistic $s_{\mathrm{y} / \mathrm{x}}$ as an estimate of the respective standard deviation [18]. For the determination of chloride, the results obtained by the MSFIA system were compared to those provided by the Mohr titration [19] and the practical detection limit was taken as the concentration at the point of intersection of the extrapolated linear segments of the calibration graph [20]. For both systems, the repeatability was assessed from 5 consecutive injections of each sample and the determination frequency was calculated from the summation of the time taken for all steps in the analytical cycle.

When the figures of merit obtained were compared, the limit of detection was lower for both determinations using volume-based sampling while the sensitivity for the spectrophotometric determination was higher when this strategy was applied. Those features were obtained as the exact volume delivered by configuration $\mathrm{A}$ is larger than that provide by configuration $\mathrm{C}$. This situation did not affect the sensitivity in the potentiometric determination.

For the spectrophotometric determination of phenolics, the results obtained for the samples analyzed were similar for both configurations and comparable to the certified value for the reference material. In the potentiometric determination of chloride, larger deviations were found, especially when configuration A was applied. When the results for repeatability were compared, there is no evidence that one of the configurations can present better results than the other.

The determination frequency was higher for time-based sampling (configuration C). Nevertheless, the time taken for sample change was not taken into account. Therefore, for

Table 5

Figures of merit for the determination of chloride using volume-based (A) and time-based (C) strategies for sample introduction

\begin{tabular}{lll}
\hline & Configuration A & Configuration C \\
\hline Calibration parameters & $E=-53.2 \pm 0.5 \log \left[\mathrm{Cl}^{-}\right]+271 \pm 1, R=-0.9993$ & $E=-53.0 \pm 0.4 \log \left[\mathrm{Cl}^{-}\right]+275 \pm 1, R=-0.9993$ \\
Limit of detection $\left(\mathrm{mg} \mathrm{l}^{-1}\right)$ & 2.3 & 3.5 \\
Determination frequency $\left(\mathrm{h}^{-1}\right)$ & 54 & 62 \\
Repeatability (RSD, $n=5)$ & $<3.3 \%$ & $<1.8 \%$ \\
Application to sample analysis (concentration in $\left.\mathrm{mg} \mathrm{l}^{-1}\right)$ & \\
Mineral water $(9.9 \pm 0.2)$ & $9.1 \pm 0.1$ & $8.1 \pm 0.1$ \\
Wastewater $(161 \pm 1)$ & $174 \pm 4$ & $158 \pm 2$ \\
Wastewater $(18.7 \pm 0.2)$ & $24.7 \pm 0.3$ & $17.0 \pm 0.2$ \\
\hline
\end{tabular}

All concentration values are expressed in $\mathrm{mg}^{-1}$. 
the reasons stated in the Section 3.1, the sampling frequency for configuration $\mathrm{A}$ would be equal to the determination rate while the value for configuration $\mathrm{C}$ would be lower than that presented in Tables 4 and 5, depending on the time and number of steps required for sample change.

\section{Conclusions}

Flow injection systems are based in the reproducible insertion of a sample plug into a flowing stream. From the results gathered in the present work, this requirement was fulfilled for all configurations tested for sample introduction in MSFIA.

Nevertheless, when the exact volume to be delivered may be important, there are some aspects to be considered, namely the internal volume of the devices (valves and confluence) used in the volume-based approach. Furthermore, the order of steps in the analytical cycle was of crucial importance when performing time-based sampling. In the present work, it was shown that any alteration in the flow direction affected the volume delivered in the subsequent step. Therefore, the introduction of a "dummy" step is recommended whenever the flow direction is changed before the sampling step.

There was no evidence that the use of either volume or time-based sampling would improve the analytical features of the determinations tested. When comparing these analytical features, some differences were found in the sensitivity for the spectrophotometric determination and in the limit of detection for both determinations. They were probably due to the differences found in the exact volume delivered by the two tested configurations and not caused by the way in which the sample was introduced in the flow system.

\section{Acknowledgements}

M.I.G.S. Almeida thanks FCT and FSE (III Quadro Comunitário de Apoio) for the Ph.D. grant SFRH/BD/8541/2002.
Prof. Víctor Cerdà is also acknowledged for helpful discussion and kind interest.

\section{References}

[1] V. Cerdà, J.M. Estela, R. Forteza, A. Cladera, E. Becerra, P. Altimira, P. Sitjar, Talanta 50 (1999) 695.

[2] M. Miró, V. Cerdà, J.M. Estela, Trac-Trends Anal. Chem. 21 (2002) 199

[3] V. Cerdà, J. Flow Injection Anal. 20 (2003) 203.

[4] F. Albertús, A. Cladera, E. Becerra, V. Cerdà, Analyst 126 (2001) 903.

[5] F. Albertús, B. Horskotte, A. Cladera, V. Cerdà, Analyst 124 (1999) 1373.

[6] M. Miró, A. Cladera, J.M. Estela, V. Cerdà, Anal. Chim. Acta 438 (2001) 103 .

[7] M.A. Segundo, A.O.S.S. Rangel, A. Cladera, V. Cerdà, Analyst 125 (2000) 1501

[8] N.V. Semenova, L.O. Leal, R. Forteza, V. Cerdà, Anal. Chim. Acta 486 (2003) 217.

[9] N. Pizà, M. Miró, J.M. Estela, V. Cerdà, Anal. Chem. 76 (2004) 773.

[10] J. Ruzicka, E.H. Hansen, Flow Injection Analysis, 2nd ed., Wiley, New York, 1988.

[11] H.M. Oliveira, Master thesis, Universidade do Porto, Oporto, 2004.

[12] A. Andrade-Eiroa, J.A. Erustes, R. Forteza, V. Cerdà, J.L.F.C. Lima, Anal. Chim. Acta 467 (2002) 25.

[13] I.M.P.L.V.O. Ferreira, J.L.F.C. Lima, L.S.M. Rocha, Fresenius J. Anal. Chem. 347 (1993) 314

[14] J. Ruzicka, E.H. Hansen, Anal. Chim. Acta 145 (1983) 1.

[15] G. Armas, M. Miró, J.M. Estela, V. Cerdà, Anal. Chim. Acta 467 (2002) 13.

[16] C. Pons, R. Forteza, V. Cerdà, Anal. Chim. Acta 424 (2004) 79.

[17] G. Armas, M. Miró, A. Cladera, J.M. Estela, V. Cerdà, Anal. Chim. Acta 455 (2002) 149.

[18] J.C. Miller, J.N. Miller, Statistics for Analytical Chemistry, 3rd ed., Ellis Horwood, Chichester, 1993.

[19] L.S. Clesceri, A.E. Greenberg, A.D. Eaton (Eds.), Standard Methods for the Examination of Water and Wastewater, 20th ed., American Public Health Association, Washington, 1998.

[20] G.G. Guilbault, Pure Appl. Chem. 53 (1981) 1907. 